\title{
DEVELOPMENT OF COMBUSTION RESPONSE FUNCTIONS IN A SUBSCALE HIGH-PRESSURE TRANSVERSE COMBUSTOR
}

\author{
M. Wierman, B. Pomeroy, and W. Anderson \\ Purdue University \\ West Lafayette, IN 47906, USA
}

\begin{abstract}
Combustion response functions describe the magnitude and time lag behavior of a flame in response to unsteady pressure and velocity. By understanding the feedback between unsteady flowfields and heat release, the growth and decay of combustion instability can be better predicted. An automated data isolation and reduction method has been developed to generate meaningful graphical combustion response functions from a combination of pressure amplitude and various image analysis metrics. It was developed and tested using pressure measurements and high-speed imaging of combustion light taken from a single element at the midspan of an unstable high-pressure subscale transverse combustor. The code was used to isolate time slices of near stationary pressure amplitude and to process the corresponding images into combustion response approximated by aggregate intensity, intensity weighted spatial center, Proper Orthogonal Decomposition (POD), and Dynamic Mode Decomposition (DMD). Overall, the generated combustion response functions generally agreed with expected behavior of an element located at a first width $(1 \mathrm{~W})$ velocity antinode and second width $(2 \mathrm{~W})$ pressure antinode. Results from both POD and DMD successfully isolated the prominent spatial and temporal light emission behavior.
\end{abstract}

\section{INTRODUCTION}

Combustion instability is the one of the most prominent unsolved problems in rocket propulsion. The coupling of unsteady heat release and chamber acoustics leads to pressure/velocity resonance $[1,2]$. This behavior is common in hightemperature, high-energy density combustion devices like gas turbines and rocket engines. Sufficiently powerful resonance increases heat release to chamber walls and injectors, leading to engine degradation and destruction. Design analysis and proof testing of engines are only possible at full scale. Past experience with 
instability has led to complete avoidance of all unstable behavior, such that the standard is that all engines must dampen all organized pressure oscillations under instability bombing (CPIA 655). Present new engine development relies on successful past designs without necessarily understanding the reasons for past success. The history of failure in new engine testing and lack of understanding for successful engine programs instill a large risk to innovate.

There is a need for physics-based understanding of acoustic and heat-release behavior that allows identification of parameters and interactions necessary for triggering, growth, and limit cycle maintenance of unstable behavior. While not as accurate as computational fluid dynamic (CFD) modeling, engineering level models that are based on the linearized Euler equations or acoustics, require orders of magnitude less time and expense and can characterize the general operation of a whole combustor. These computations require input of physically meaningful combustion response models that could be generated from experimental or high fidelity CFD results $[3,4]$. It also necessitates more rigorous means of comparison and validation between experiments and computations. The process elements establish a cycle of modeling and validation that could produce a robust a priori predictive model of combustion instability for rockets and aeropropulsion systems.

This paper addresses techniques that are being developed to study interaction between unsteady heat release and gas dynamics that drives the initiation and growth of combustion instability. First, the subscale combustor used to provide the means to measure combustion response of the reacting flow of a study injector element to varying amplitude levels of transverse oscillations is described. Highspeed imagery is used to record temporally and spatially resolved $\mathrm{CH}^{*}$ emission from a study element as an indicator of heat release, and a series of analytical techniques that are used to reduce these large data sets into graphical response functions are described. To demonstrate the approach, the combustion response of a gas-centered, swirl coaxial injector element to varying levels of pressure and velocity oscillations is calculated, and results from the different methods are compared.

\section{EXPERIMENTAL METHOD}

\subsection{Flame Describing Functions}

Nonlinear flame response models are constructed to address the functional relationship between unsteady heat release and global or local perturbations of pressure and velocity $[5,6]$. Combustion response transfer functions, or flame describing functions (FDF), describe the magnitude and phase of heat release as a function of local unsteady pressure/velocity. They have been constructed 
from measurements of pressure/velocity and $\mathrm{CH}^{*}$ chemiluminescence, used to approximate heat release [7]. Low-amplitude fluctuations tend to have a linear response in magnitude, which concurs with the use of linear input growth rates in classical models $[5,8]$. This approach simplifies computation by taking a solely linear approach. However, the combustion response at high-amplitude fluctuations reaches a saturation level, which (in conjunction with increased acoustic damping) allows the development of limit cycle behavior. The shift to nonlinear behavior has generally been linked to unsteady amplitude, Reynolds number, equivalence ratio, and forcing frequency [7].

Hield et al. treated gain and phase as functions of local amplitude and fitted them to an exponential curve to give a rudimentary nonlinear functional form [7]:

$$
\begin{gathered}
\frac{q^{\prime}(t)}{\bar{q}}=\frac{n\left(u_{\mathrm{rms}}\right)\left(u^{\prime}\left(t-\tau\left(u_{\mathrm{rms}}\right)\right)\right)}{\bar{u}} ; \\
n, \tau \sim c+a e^{b u_{\mathrm{rms}}}
\end{gathered}
$$

where $n$ is the gain; $\tau$ is the phase lag; $q$ is the heat release; $u$ is the velocity; and $a, b$, and $c$ are the empirically determined constants. Terms that are prime and bar represent perturbation and mean quantities, respectively. $n$ and $\tau$ were specifically mapped against root mean square (rms) velocity rather than the instantaneous oscillating velocity [7]. The strength of the measured correlation suggests combustion response follows amplitude rather than instantaneous velocity/pressure. Spectral content was greater at lower frequencies, suggesting that the combustion response acts roughly as a low pass filter [9]. By coupling this low pass filter-like combustion response with Culick's Galerkin model of gas dynamics and response, it is hypothesized that the combustion would significantly drive only lower modes, and higher modes would primarily arise due to energy transfer between modes [10].

Flame describing functions are typically determined empirically from experiments and are not fit to any prescribed physical model (Fig. 1). Given the diversity of injector designs, flow conditions, and combustion characteristics, it is unlikely a generalized form could be developed that would apply to different experiments. For a priori prediction, these empirical models shall either have to be matched to a physics-based model or have predictive behavior drawn from a yet-to-be-measured array of baseline injector data. In the interests of injector design possibilities and considering the potential size of such a database, a mechanistic description of injector physics will be most useful for future design efforts, even if such a model is not immediately clear at this time.

Largely due to current methods of computation and experimental measurement, present models are only used for discrete or global spatial effects. Highspeed imaging allows both temporally and spatially resolved measurements of the signal from combustion light and is leading to improved understanding of the spatial distribution of the combustion as a function of the unsteady amplitude. 


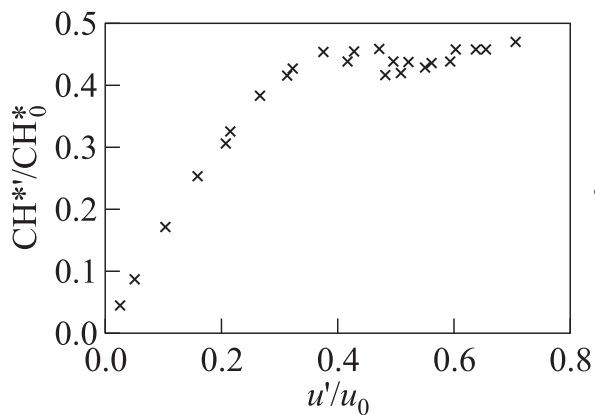

(a)

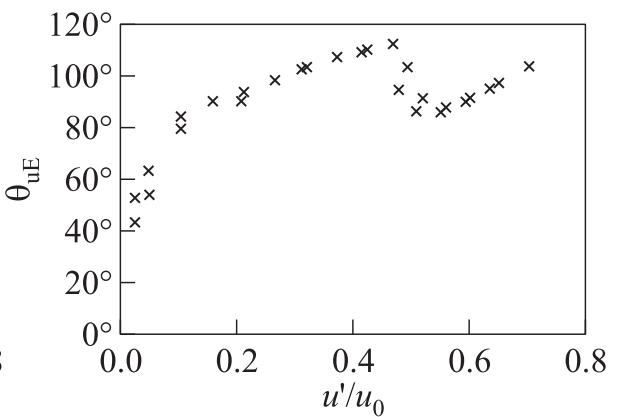

(b)

Figure 1 Example flame describing function showing gain and phase of combustion response as a function of normalized unsteady velocity [5]

\subsection{Transverse Combustor}

The transverse combustor (Fig. 2) is a rectangular combustor comprising a linear array of seven ox-centered, fuel swirl elements injecting decomposed $\mathrm{H}_{2} \mathrm{O}_{2}$ and JP-8 at chamber pressures of $\sim 860$ to $\sim 975 \mathrm{kPa}$ depending on the injector configuration $[8,9]$. Unsteady peak-to-peak pressure amplitudes similarly range from $11 \%$ to $72 \%$ of measured chamber pressure with varying degrees of amplitude

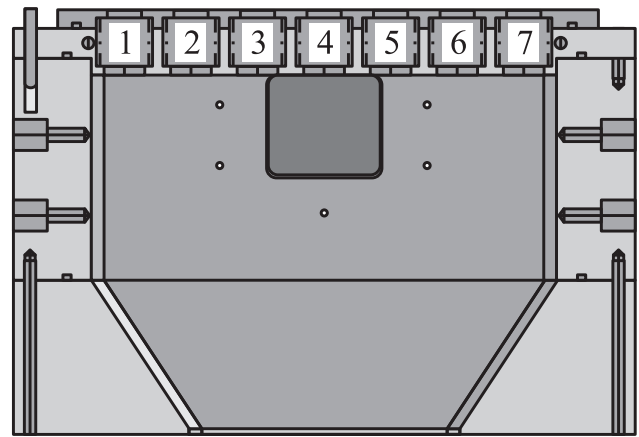

(a)

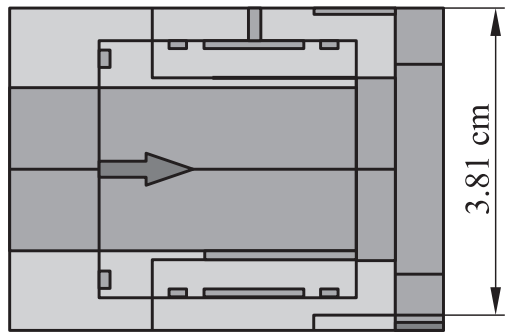

(b)

Figure 2 (a) A subscale transverse combustor. Elements are ox-centered shear coaxial injectors using JP-8 and decomposed $\mathrm{H}_{2} \mathrm{O}_{2}$. The chamber width is $26.7 \mathrm{~cm}$. The chamber length including the nozzle is $23.5 \mathrm{~cm}$. The chamber height is $3.81 \mathrm{~cm}$. The window is placed at the center of the chamber and adjacent to the injector face to permit visibility of the flow and combustion light emission of the center study element. (b) The study element (Element 4) is a gas-centered swirl coaxial element located at the velocity antinode of the $1 \mathrm{~W}$ mode, which is driven by the six outer elements [8] 
Table 1 Operating conditions

\begin{tabular}{|c|c|c|c|c|c|c|c|c|c|c|}
\hline & \multirow[b]{2}{*}{$\begin{array}{l}P_{c}, \\
\mathrm{kPa}\end{array}$} & \multirow[b]{2}{*}{$\mathrm{O} / \mathrm{F}$} & \multicolumn{3}{|c|}{$\dot{m}, \mathrm{~kg} / \mathrm{s}$} & \multicolumn{2}{|c|}{ Total $p^{\prime}$} & \multirow{2}{*}{$\begin{array}{c}1 \mathrm{~W} \\
\text { frequency, } \\
\mathrm{Hz}\end{array}$} & \multicolumn{2}{|c|}{ Injector configuration } \\
\hline & & & Oxid & $\begin{array}{c}\text { Driving } \\
\text { fuel }\end{array}$ & $\begin{array}{c}\text { Study } \\
\text { fuel }\end{array}$ & $\mathrm{Pa}$ & $\%$ & & $\begin{array}{c}\text { Mono- } \\
\text { propellant }\end{array}$ & $\begin{array}{c}\text { Bi- } \\
\text { propellant }\end{array}$ \\
\hline$\overline{\mathrm{LOV}}$ & 864 & 14.55 & 1.3 & 0.064 & 0.030 & 95 & 11 & 1850 & $1,3,5,7$ & 2,4 \\
\hline Med & 855 & 14.35 & 1.360 & 0.064 & 0.030 & 340 & 40 & 1800 & $2,3,5,6$ & $1,4,7$ \\
\hline High & 975 & 8.37 & 1.355 & 0.132 & 0.030 & 700 & 72 & 2030 & 3,5 & $1,2,4,6,7$ \\
\hline
\end{tabular}

steadiness. This modulation is achieved by selecting elements as monopropellant or bipropellant. Measured pressure signals show highly nonlinear behavior with steep-fronted pressure waveforms.

Depending on the injector configuration, the $1 \mathrm{~W}$ mode ranges from $\sim 1750$ to $\sim 2000 \mathrm{~Hz}$ with higher harmonics at whole number multiples of the fundamental frequency. Past testing has selectable conditions of unstable pressure amplitude [9]. This was achieved by setting specific injector elements as monopropellant or bipropellant. Settings labeled as Low, Medium, and High are achieved using different injector configurations, as detailed in Table 1. In general, increasing pressure amplitude was achieved with increasing fuel input and with moving fuel injection closer to chamber walls.

Optical accessibility is provided to the study element through a flat quartz window which allows good transmission of light in the emission range of the $\mathrm{CH}^{*}$ radical $(430 \mathrm{~nm})$ [10]. Images of $\mathrm{CH}^{*}$ intensity are recorded with a Phantom V7.1 black and white high-speed camera, a Semrock 434-nanometer narrow band filter, and a Videoscope VS4-1845HS intensifier. The observed study element is located at the measured $1 \mathrm{~W}$ velocity antinode and a $2 \mathrm{~W}$ pressure antinode.

\subsection{High-Speed Image Analysis}

High-speed movies contain significant quantities of data. A single test with a black and white $256 \times 256$ pixel frame recorded at 20,000 frames per second for $0.75 \mathrm{~s}$ generates $2.7 \mathrm{~GB}$ of data. Meaningful processing of such large quantities of data from a full test series requires automated, orderly computational processing to properly analyze and isolate significant behaviors. Various analytical techniques have been investigated for such processing with varying levels of spatial and temporal resolution. The method used should be based on the specific objective and fidelity of the analysis.

\subsubsection{Mean Image}

The mean image is the pixel-by-pixel time-averaged signal of light intensity. It produces a single frame with no time-dependent data. The mean combustion 


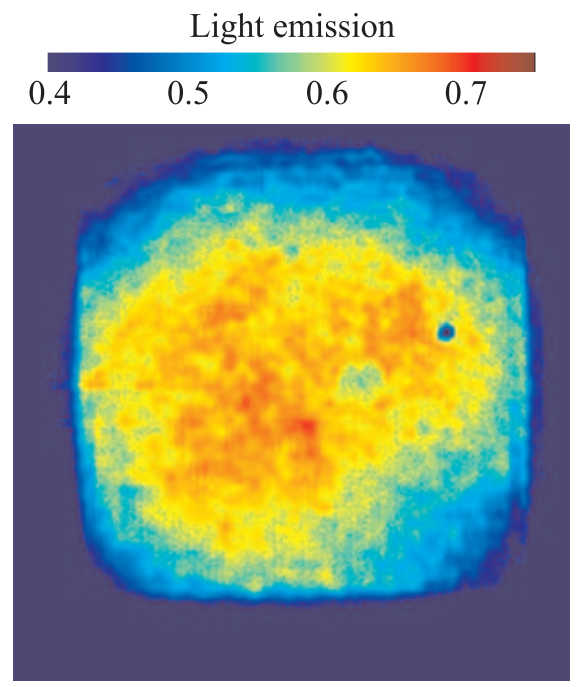

Figure 3 Time-averaged filtered combustion light emission of the center element in the transverse chamber shown in Fig. $2 a$. The window is 9.53-centimeter square. The injector is at the top center of the frame. Flow is top to bottom. The small blue spot in center right is a window imperfection

light distribution and intensity from a typical test are displayed in Fig. 3. This is useful in qualitatively showing the combustion distribution during a given time slice and establishes a normalization value for later perturbation calculations:

$$
\bar{I}(x, y)=\frac{1}{n} \sum_{i=1}^{n} I(x, y, t(i)) .
$$

\subsubsection{Aggregate Intensity}

The Aggregate Intensity method reduces each frame to a single value by finding the average pixel value for each frame. This produces a one-dimensional (1D) vector of average pixel value as a function of time. While this does reduce spatial resolution to a single value representing the entire viewing area of the window, Aggregate Intensity is a simple metric to demonstrate overall light intensity perturbations and has been used to develop flame describing functions $[7,9]$. This is especially useful in studying pressure-coupled effects due to their scalar (nondirectional) nature. When normalization is desired, it is best to normalize Aggregate Intensity by the mean pixel intensity of the mean frame:

$$
I^{\prime}(t)=\sum_{x} \sum_{y}(I(x, y, t)-\bar{I}(x, y)) .
$$




\subsubsection{Center of Intensity}

The Center of Intensity method finds the center of each frame in $x$ - and $y$-coordinates weighted by the perturbed pixel value. This is mathematically similar to finding a center of mass, where the pixel intensity value is used in place of the mass. As the whole flame is perturbed in a single direction, the center of intensity for that direction will be perturbed as well. This is especially useful in studying velocity response. The flame itself may not change in total intensity, but the shifting location of the flame will shift the intensity-weighted $x$ and/or $y$ center of intensity. When normalization is desired, use the height or width of the window or the chamber in appropriate units. For processing of high-speed camera movies, convert all length scales to pixels:

$$
\begin{aligned}
X^{\prime}(t) & =\frac{\sum_{x} \sum_{y}(x I(x, y, t))}{\sum_{x} \sum_{y} I(x, y, t)} ; \\
Y^{\prime}(t) & =\frac{\sum_{x} \sum_{y}(y I(x, y, t))}{\sum_{x} \sum_{y} I(x, y, t)} .
\end{aligned}
$$

\subsubsection{Proper Orthogonal Decomposition}

Proper orthogonal decomposition is a method to obtain low-dimensional approximations of high-dimensional phenomena. Proper orthogonal decomposition seeks to decompose an input into a series of modes of the form:

$$
z(x, t) \approx \sum_{k=1}^{M} s_{k} a_{k}(t) \varphi_{k}(x)
$$

where $z$ is the input matrix with spatial and temporal behavior; $s_{k}$ is the singular value or "energy;" $a_{k}$ is the temporal component; $\varphi_{k}$ is the spatial component; and $M$ is the desired number of modes of approximation. As $M$ tends to the number of raw input samples, the input matrix is reconstructed from these modal components.

In a full POD, an input matrix is decomposed into pairs of orthonormal row and column eigenvectors, which represent associated spatial and temporal components. In this manner, the different eigenvector pairs are independent and contain unique information (orthogonal). The associated eigenvalue of each pair represents the singular value, or "energy." Each set of a single spatial mode, temporal mode, and singular value represents a single POD mode, which is a basis independent from all other POD modes. In this decomposition, the input information is sorted into these mode sets by decreasing order of POD mode energy, which represents the magnitude of variation and influence captured by 
each POD mode. Recomposing all the POD modes will reconstitute the input data $[11]$.

For discrete data sets, the POD approximation is of the form:

$$
z_{M}=\sum_{k=1}^{M} U_{k} S_{k} V_{k}^{T}
$$

where $z_{M}$ is the best $M$-term approximation of the input matrix; $S_{k}$ is the singular value or "energy;" $U_{k}$ is the temporal component; and $V_{k}$ is the spatial component. For matrix input, $M$ is limited by the smaller of the matrix height and width. For high-speed movies, these correspond to the total number of pixels and the number of frames.

The decomposition was performed using the Matlab svds command, which performs the singular value composition on a two-dimensional (2D) input matrix for a given number of output modes $(M)$. The mean image is effectively the 0 th order approximation of the system and has a constant effective temporal component of one for the full movie length. Analysis resulting in the first POD mode comprising a mean spatial image with a varying temporal component violates the definition of the mean as time-invariant; in the interests of analyzing only perturbations, the mean image was calculated and then subtracted from each frame; so, the POD modes include only time-dependent spatial modes.

The frames were then converted into single row vectors and concatenated in temporal order to produce the $2 \mathrm{D}$ input matrix for the POD. This process converts a three-dimensional (3D) array into a $2 \mathrm{D}$ matrix with dimensions of space and time. The conversion was done in reverse on the POD spatial component output $(V)$ to produce 2D spatial component matrices of the same dimensions as the input frames.

As seen in Fig. 4, a POD mode provides a description of the linked spatial and temporal character associated with a singular value, or "energy." The spatial mode corresponds to the visible region of the combustor, showing magnitude and phase between different regions of combustion. The combustion on the left and right sides of the combustor is out of phase by $180^{\circ}$ (i. e., opposite sign).

The associated temporal component has frequency content denoted by its power spectral density (PSD), indicating primary content at $2032 \mathrm{~Hz}$. These correspond to the 1st unstable mode as measured by high-frequency pressure transducers. Without prior input of user-desired content, the POD has isolated mode(s) which match unstable signals in the combustor. In this case, sufficiently powerful acoustic resonance will be the primary effect on combustion in these subscale experiments. This modal isolation fits the requirement of POD to produce low-dimensional approximations of high-dimensional effects.

When normalization is desired, the temporal component vector should be multiplied by its energy and the rms of its spatial component and divided by the mean pixel intensity of the mean frame. This ensures that a 1D time trace 


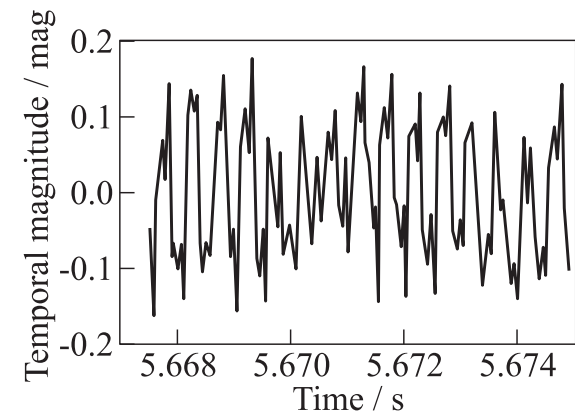

(a)

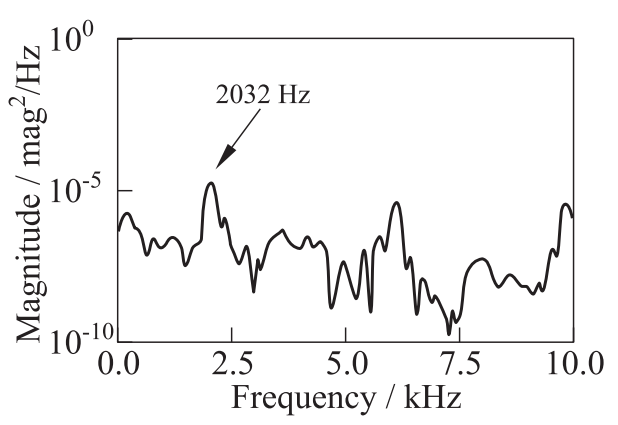

(b)

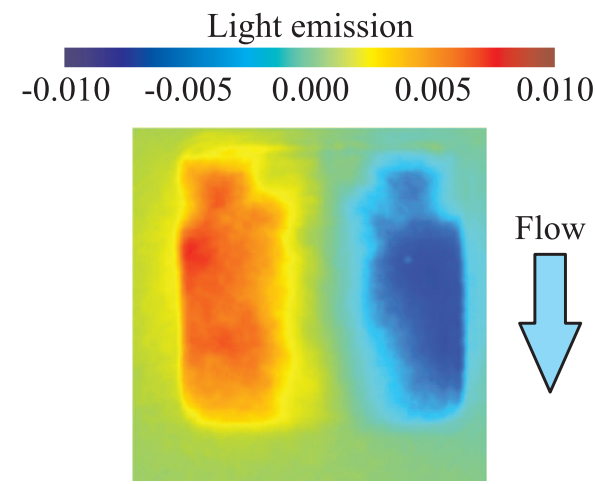

(c)

Figure 4 The 1st POD mode from the subscale transverse rocket combustor in highamplitude configuration $\left(p^{\prime} \sim 0.7 p_{c}\right)$ : $(a)$ the temporal component; $(b)$ the PSD of the temporal component, showing most notable frequency content at $2032 \mathrm{~Hz}$, which matches the 1st acoustic mode from the measured pressure signal; and $(c)$ the spatial component. Flow is from top to bottom and the injector is at the top of the frame and located at the expected $1 \mathrm{~W}$ velocity antinode. The positive and negative regions on the right and left, respectively, indicate that combustion light emission alternates sign between the sides of the injector. Coupled with the periodic character measured in the PSD, it is evident that the combustion is strongly affected by the local perturbations, specifically, velocity fluctuations of the 1st acoustic mode

is maintained and all relevant components are matched and normalized against an aggregate average metric.

\subsubsection{Dynamic Mode Decompositon}

Dynamic mode decomposition is similar to POD in that it decomposes data into orthogonal bases of related temporal and spatial components. Where POD 
sorts modes based on an "energy" value derived from the magnitude of the most descriptive behaviors, DMD sorts modes on the basis of frequency. Proper orthogonal decomposition modes are able to represent multiple frequencies, while each DMD mode is tied to a single frequency. For input data of significant frequency content, DMD is especially useful for isolating individual acoustic mode behavior, which is often linked to specific frequencies [12-16].

$$
z(x, t)=\sum_{k=1}^{N} a_{k}(t) \varphi_{k}(x)
$$

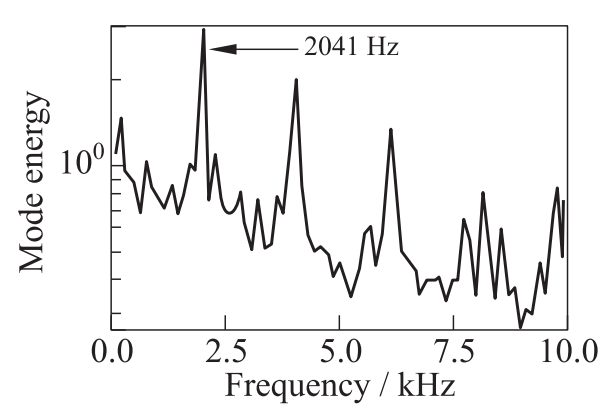

(a)

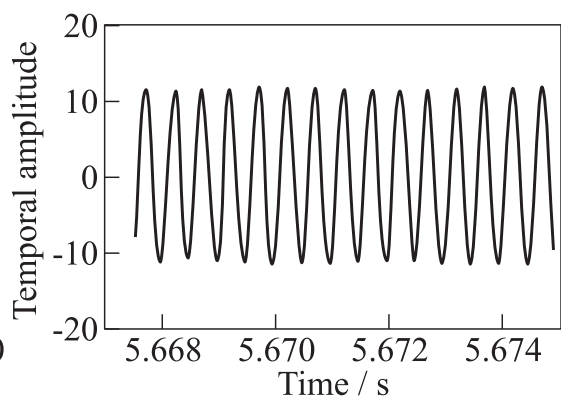

(b)

Light emission

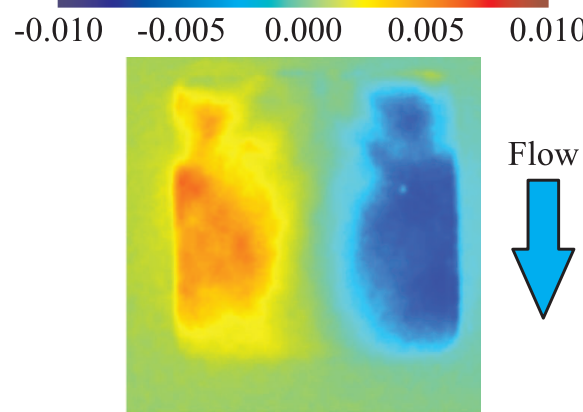

(c)

Figure 5 The DMD mode of $2041 \mathrm{~Hz}$ from the subscale transverse rocket combustor. This frequency has the largest associated mode energy (as seen in $(a)$ ) and matches the 1st acoustic mode from the measured pressure signal; $(b)$ the temporal component; and $(c)$ the spatial component. Coupled with the periodic temporal signal, the positive and negative regions on the right and left, respectively, indicate that combustion light emission alternates sign between the sides of the injector. Both the 2041-hertz DMD mode and the 1st POD mode show similar spatial and temporal behavior and are the most prominent behavior from their respective analyses 
where $z$ is the input data; $a_{k}$ is the temporal component; $\varphi_{k}$ is the spatial component; and $N$ is the total number of modes. In this calculation, the temporal component carries the units and intensity magnitude, and the spatial component is normalized. Approximation can be performed by selecting specific DMD modes to go into the sum.

In DMD, an input matrix is decomposed into pairs of orthonormal row and column eigenvectors, which represent associated spatial and single frequency temporal components. In this manner, the different eigenvector pairs are independent and contain unique information (orthogonal). In this decomposition, the input information is sorted into these mode sets by increasing value of temporal mode frequency energy. Recomposing all the DMD modes will reconstitute the input data.

As with POD, the mean image was calculated and then subtracted from each movie frame. The frames were then converted into single row vectors and concatenated in temporal order to produce the 2D input matrix for the DMD. This process converts a $3 \mathrm{D}$ array into a $2 \mathrm{D}$ matrix with dimensions of space and time. The conversion was done in reverse on the DMD spatial component output to produce $2 \mathrm{D}$ spatial component matrices of the same dimensions as the input frames.

As seen in Fig. 5, DMD isolates behavior based on frequency. The mode energy distribution over frequency shows most prominent behavior at 2041, 4080, and $6120 \mathrm{~Hz}$, which correspond exactly to the $1 \mathrm{~W}, 2 \mathrm{~W}$, and $3 \mathrm{~W}$ pressure modes, respectively. The 2041-hertz temporal and spatial components are very similar to POD Mode 1 from Fig. 4 as velocity coupling at the first acoustic mode is the most prominent unsteady behavior. Although DMD and band-pass filtering may produce similar results, it should be noted that they are fundamentally different; during bandpass filtering, data are necessarily lost through filtering windows, whereas DMD is a true decomposition and all input data can be recomposed from the full set of DMD modes if desired.

When normalization is desired, the temporal component vector should be multiplied by the single value rms of its spatial component and divided by the mean pixel intensity of the mean frame.

\subsection{Combustion Response Function Development}

It has already been stated that combustion response is amplitude- and frequencydependent. For combustion response function development from large data sets, selection by hand of relevant data slices where the pressure signal is sufficiently stationary is both time consuming and subjective. It is necessary to have an accurate and automated process to isolate data sets for analysis. The present code takes inputs of pressure data and cine format movies from multiple tests and determines combustion response functions based on several image analysis metrics at selected data slices. 


\subsubsection{Slice Selection}

Slices of pressure and movie data are selected using an automated process. From a raw input pressure signal, the time period of overlap between the pressure signal and the movie data is analyzed for pressure frequency content through a PSD. The most powerful PSD peak near an input frequency guess is selected as the frequency of analysis. In this analysis, the guess is the fundamental frequency of the combustor. The overlapped pressure signal is bandpass-filtered around the analytically chosen frequency $( \pm 5 \%)$. Starting at the beginning of the pressure signal, data slices of selected length are analyzed to find the standard deviation of the amplitude envelope normalized by the mean amplitude of the data slice. If this value is below a specified value, the slice timestamps are recorded, and analysis continues to the next full specified length. If this condition is not met, the window moves a single period forward. This continues until the entire filtered signal has been processed. The analytical window is set at 15 periods of the computationally chosen frequency, and the normal-

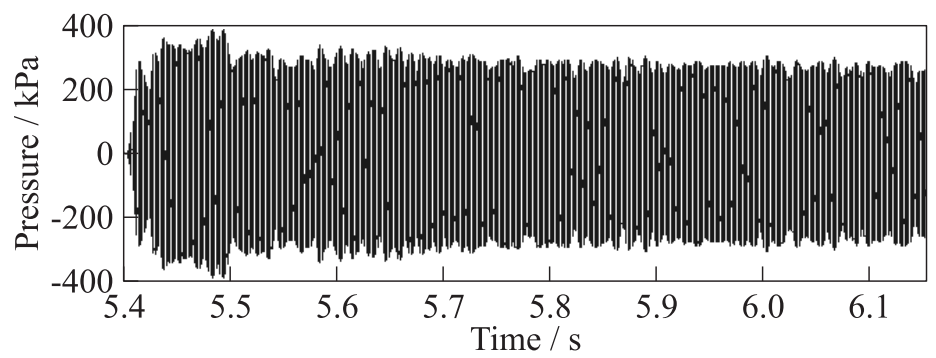

(a)

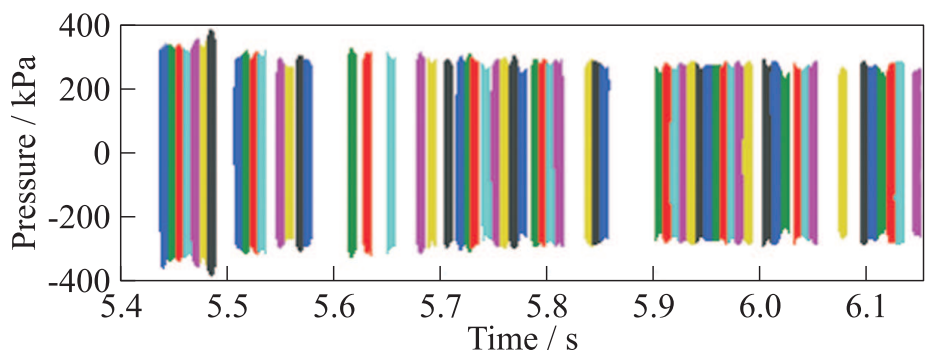

(b)

Figure 6 Slice selection from a test in high-amplitude $\left(p^{\prime} / p_{c} \sim 0.7\right)$ configuration: (a) pressure signal as a function of time from a side wall high-frequency pressure transducer bandpass filtered around $2025 \mathrm{~Hz}$, the computationally chosen most powerful frequency; and (b) 61 slices of 15 periods each that are within the specified normalized amplitude deviation of 0.03 
ized amplitude standard deviation limit is set at 0.03 . These are arbitrary values chosen to yield steady amplitudes over sufficient slice lengths to show steady behavior while allowing for an analytically meaningful number of possible slices.

The start and end time stamps of the data slices are used to isolate pressure and cine movie data. This process is automated to analyze several tests in sequence, resulting in a series of data slices of unsteady pressure and light emission at near-steady pressure amplitude as seen in Fig. 6 . The cine movie data is then converted into Matlab data and processed using the techniques previously specified (Aggregate Intensity, $x$ and $y$ centers of intensity, POD, or DMD). Other techniques can be easily added for future processing.

\subsubsection{Response Function Generation}

The next step is to convert these near-steady slices of various amplitudes into individual data points to build functional relationships between pressure amplitude and image response. In this processing, rms values are calculated for the normalized values of the $1 \mathrm{D}$ temporal pressure and image response. The pressure, Aggregate Intensity, and $x, y$ centers of intensity signals are already $1 \mathrm{D}$ signals and do not need any further postprocessing before finding rms. For POD and DMD, a single temporal mode must be chosen to be processed through rms. Normalization is performed as described previously. This process reduces the 1D temporal amplitude traces from each slice into single values for pressure and image metric amplitude. The array generated in this manner is then plotted to discern functional relationships between pressure amplitude and image response. Frequency response can be seen as well by band pass filtering the pressure and image response slices for discrete slices of frequency prior to the rms calculation.

Time lag is determined for the DMD responses. The pressure data is bandpass filtered at the same frequency as the DMD mode. The cross correlation between the DMD temporal mode and the bandpass filtered pressure signal is used to determine the time lag in degrees between the two signals.

\section{RESULTS}

\subsection{Aggregate Intensity Response Function}

In Fig. 7, the Aggregate Intensity response function shows a linear rise for increasing amplitude. The frequency response shows its most significant content near $4000 \mathrm{~Hz}$, which corresponds to the $2 \mathrm{~W}$ mode. This makes sense given the studied element is located at a $2 \mathrm{~W}$ pressure antinode. 


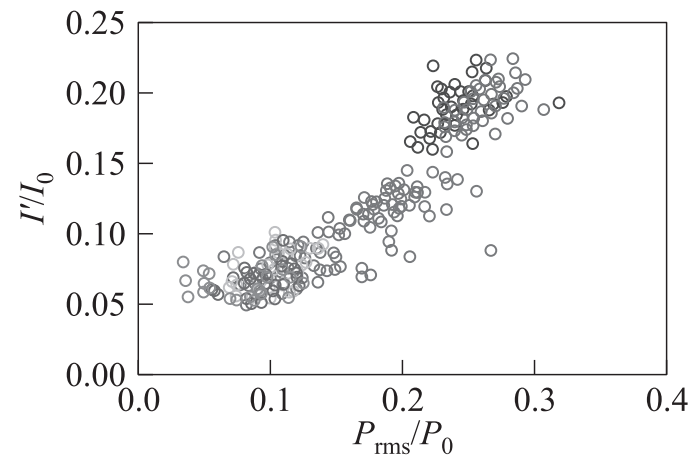

(a)

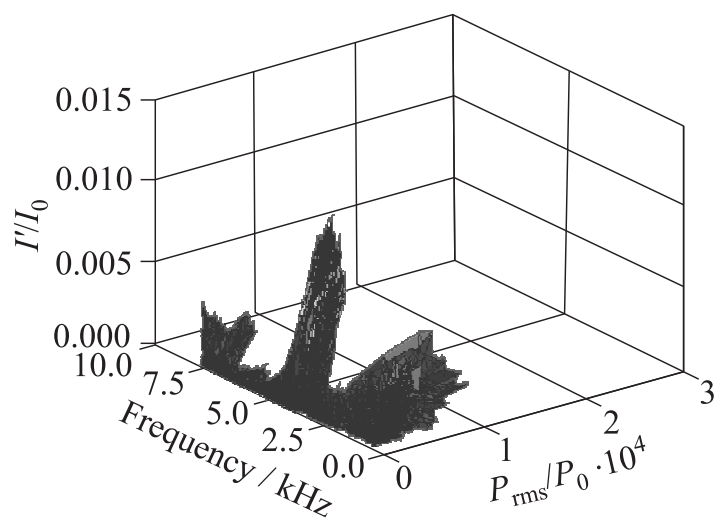

(b)

Figure 7 Response function of normalized Aggregate Intensity as a function of normalized pressure amplitude only $(a)$ and as a function of frequency and normalized pressure amplitude $(b)$ at different tests

\subsection{Center of Intensity Response Function}

In Fig. 8, the $x$ component center of intensity response function shows a split between a flat response and a linear falling response for low and medium amplitudes. The $x$ direction corresponds to the transverse direction in the combustor. These data points indicate the magnitude of transverse motion of the flame intensity center. The frequency response shows most significant content near $2000 \mathrm{~Hz}$ which corresponds to the $1 \mathrm{~W}$ mode. The study injector is located at the $1 \mathrm{~W}$ velocity antinode. Combined with the comparative lack of response in the global intensity fluctuation at the $1 \mathrm{~W}$ frequency, this suggests that the strong $1 \mathrm{~W}$ velocity perturbations shift the flame left and right but do not cause any significant fluctuations in the light emission. 


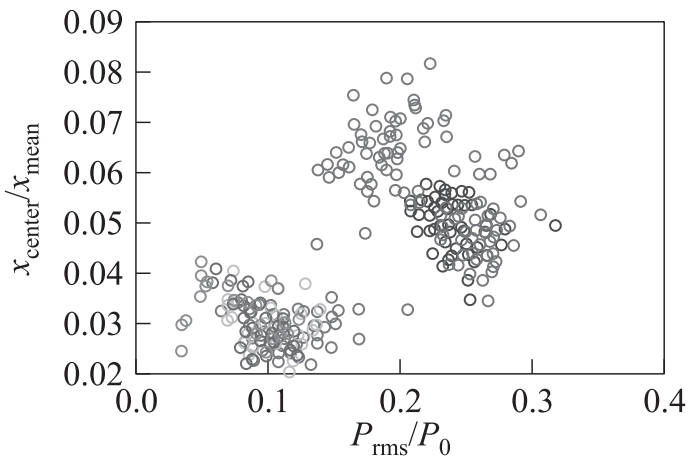

(a)

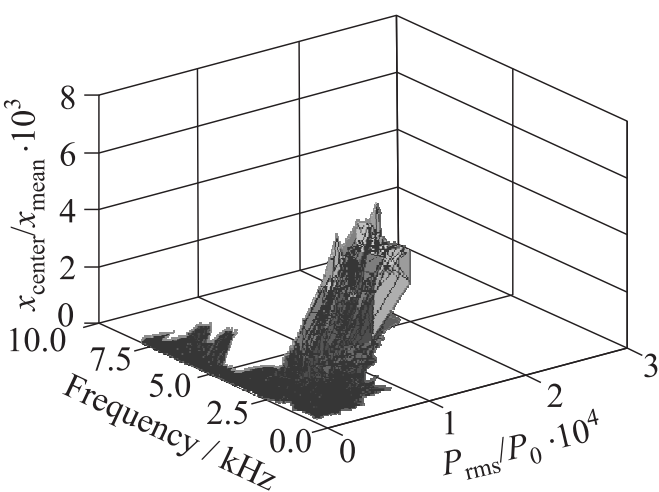

(b)

Figure 8 Response function of normalized $x$ component of the center of intensity as a function of normalized pressure amplitude only $(a)$ and as a function of frequency and normalized pressure amplitude $(b)$ at different tests

\subsection{Proper Orthogonal Decomposition Mode 1 Response Function}

In Fig. 9, the normalized POD Mode 1 response function shows a linear rising response for increasing amplitude. In all tests, POD Mode 1 showed most significant content at the $1 \mathrm{~W}$ frequency as this is the most powerful and descriptive acoustic mode.

\subsection{Dynamic Mode Decomposition Magnitude Response Functions}

In Fig. 10, the normalized DMD mode response functions show response at the two primary measured acoustic modes, which also corresponded to the strongest measured DMD modes. The $1 \mathrm{~W}$ mode response shows a clear separation in response between the low, medium, and high cases. The low-amplitude response 


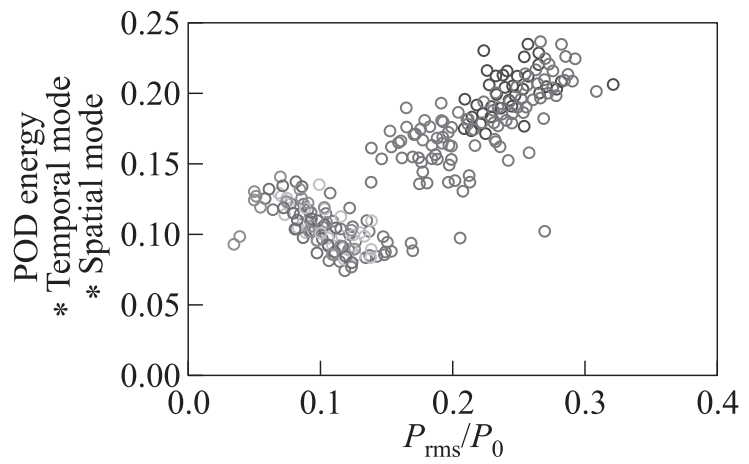

(a)

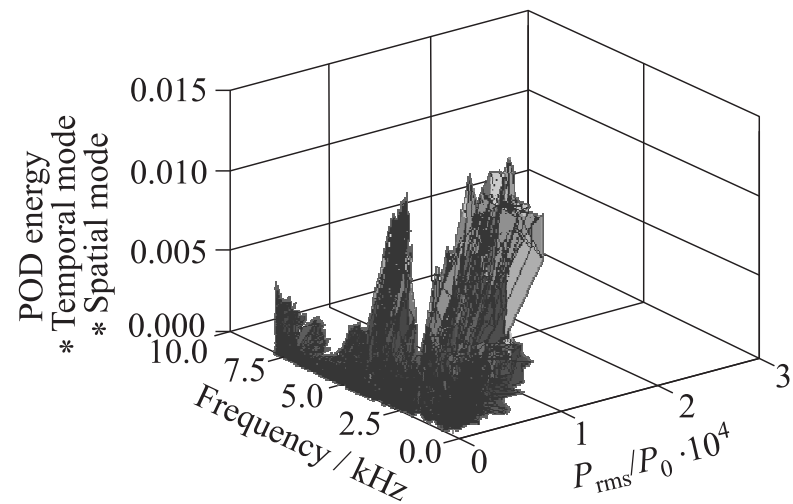

(b)

Figure 9 Response function of normalized POD Mode 1 as a function of normalized pressure amplitude only $(a)$ and as a function of frequency and normalized pressure amplitude $(b)$ at different tests

shows a decreasing linear slope, followed by a jump in medium amplitudes and a fall through increasing amplitude. The $2 \mathrm{~W}$ mode response shows a positive linear slope region for increasing pressure amplitude. The frequency isolation of DMD may explain the unique responses measured compared to other analysis techniques. The $2 \mathrm{~W}$ response shows only positive slopes while the $1 \mathrm{~W}$ response shows a negative slope in higher amplitudes. Negative slopes at higher amplitudes may be indicative of saturation at those high amplitudes, allowing more energy input to drive increasingly higher modes.

\subsection{Dynamic Mode Decomposition Time Lag Response Function}

In Fig. 11, the DMD/pressure time lag response function shows the time lag in degrees of the DMD response relative to the bandpass filtered pressure peak on 


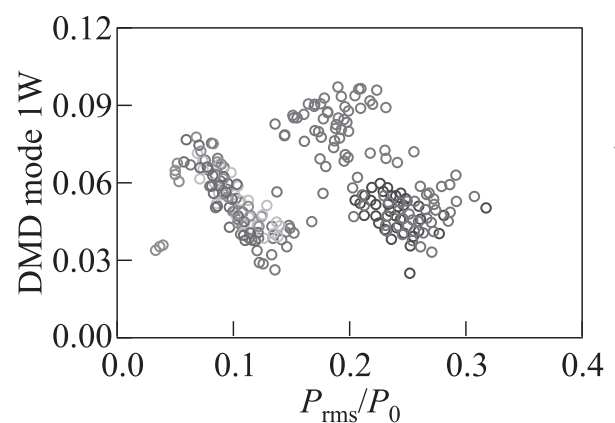

(a)

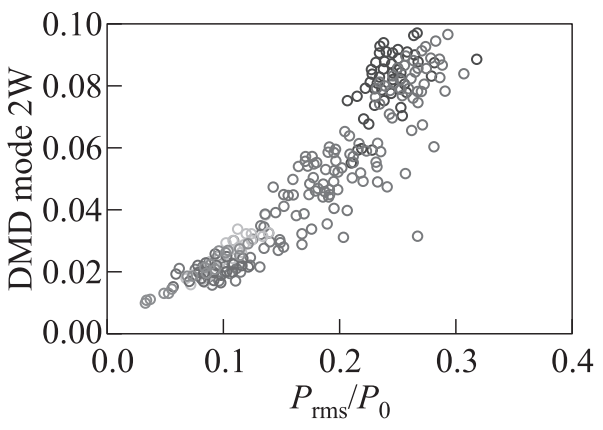

(b)

Figure 10 Response functions of normalized DMD modes at the 1W (a) and 2W (b) modes as functions of normalized pressure amplitude at different tests

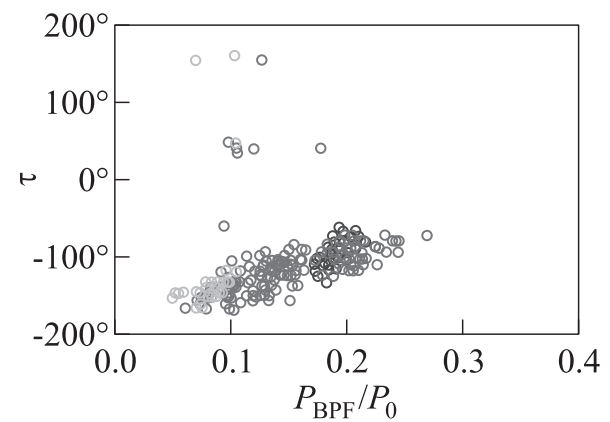

Figure 11 Response function of time lag between band pass filtered (BPF) pressure and $1 \mathrm{~W}$ DMD mode as a function of normalized pressure amplitude at different tests

the right wall of the combustor. Using this metric, the acoustic $1 \mathrm{~W}$ velocity peak at the study element in the center of the chamber will occur at $-90^{\circ}$. The $1 \mathrm{~W}$ time lag response is at the expected $-90^{\circ}$ at high pressure amplitude and linearly increases the time delay for decreasing pressure amplitude. For lower forcing amplitude, the time lag is further shifted from response immediately at the acoustic velocity peak.

\section{CONCLUDING REMARKS}

The empirical combustion response of a gas-centered, shear-coaxial injector to forced pressure and velocity oscillations is generated empirically using a variety of methods. The response functions generally fit expected behavior based on the placement of the observed element in the unstable flowfield. As a measure of the 
response in aggregate intensity perturbation, Aggregate Intensity showed a linear response for all pressure amplitudes. The $x$ center of intensity response showed a low magnitude flat response for low amplitudes and a higher magnitude falling response for high amplitudes. These two measures corresponded most strongly to the $2 \mathrm{~W}$ and $1 \mathrm{~W}$ modes, respectively. As Aggregate Intensity best captures stationary emission perturbation and center of intensity best captures motion of the flame, these responses correspond well to the expected behavior of an element at a $1 \mathrm{~W}$ velocity antinode and a $2 \mathrm{~W}$ pressure antinode. These two relatively simple methods provide some insight to instability behavior and allow a good qualitative check of processed behavior. However, the data are reduced to such a large degree that little practical application is possible towards spatially resolved distributions in engineering level models. Better resolved techniques are necessary for a better characterization of flame behavior.

More sophisticated modal decomposition techniques were also used to analyze the data. The Mode 1 POD response shows a general linear rise with increasing pressure amplitude. The DMD $1 \mathrm{~W}$ magnitude response matches the form and frequency content of the $x$ center of intensity response. The DMD $2 \mathrm{~W}$ magnitude response matches the form and frequency content of the Aggregate Intensity response. The DMD 1W time lag response indicates near immediate response with local velocity perturbations and longer response time with decreasing pressure amplitude, indicating a delay present at lower forcing.

Modal analysis through POD and DMD is a potential major improvement that naturally follows the Galerkin approach of amplitude growth arising from the spatial-temporal cross section of heat release and pressure modes. In producing a spatially and temporally resolved response, these analyses produce insight into physical behavior not possible with the other discussed techniques. In application to experimental results as well as computational results, these modal decomposition techniques are more useful in gaining insight of combustor behavior and should be used when possible because they are closer to a physics-based model. The concurrent decomposition analysis of simulation results and experimental results provides a more rigorous means of comparison and validation than do lower-order means like separately comparing frequency, amplitude, and mode shape. These latter methods could compare well but still be inaccurate because of cumulative errors. Through concurrent decomposition, experimental and computational results can be identically processed for comparative validation and insertion into low-order model databases.

Both POD and DMD show spatially and temporally resolved modes of the overall behavior. The principal contrast between the two methods is the ordering by descriptivity and frequency, respectively, of the two methods. Since POD modes often contain frequency content from multiple frequencies and DMD modes necessarily are limited to a single frequency, the choice of method is dictated by the usefulness as applied to the input data and related predictive techniques. With acoustic behavior and multiple harmonics being recorded by both 
pressure and emission measurement, DMD analysis was the preferred method for direct modal comparison and input to present models. The frequency sorted content enabled the best comparison to single frequency predictive codes, the best assignment to acoustic modal behavior, and the least reduced analytic approach to time lag determination. However, the energy sorting method of POD has use outside of the present combustion response calculation in construction of reduced order models and detection of more complex behavior not, otherwise, revealed on a frequency basis.

The present work is exploratory, and more work is required to generate better combustion response functions. In the formulation here, the intensity signal was not corrected for pressure effects. Emitter density should nominally scale directly with local pressure perturbations. Flame response and emitter density will be decoupled in future calculations. Another possible limitation was the size of the window relative to the flame size of the study element. The light emission from the study element should be captured fully in the window. However, sufficiently large flames or perturbations can disturb a flame outside the visible area and limit total light collection and spatial resolution. The DMD technique produces less errors than POD when the view area is limited.

Determination of empirical combustion response models can be complicated if the oscillating field is nonstationary; thus, an automated technique is necessary to identify time slices of near-stationarity so that reliable combustion response can be measured. Overall, the slice selection code successfully isolated a meaningful number of near steady amplitude data slices from input data taken from several tests at varying levels of pressure amplitude. The code was able to easily incorporate new methods of image analysis and determine response functions based on whole or part of the input data. It was written for ease of use for any data using unsteady pressure/velocity measurements and is being implemented in studies in longitudinal rocket and gas turbine combustion instability.

\section{ACKNOWLEDGMENTS}

These experiments were sponsored by the Purdue University Department of Aeronautics \& Astronautics. The first author is supported by the NASA Space Technology Research Fellowship (NSTRF). The second author was supported by the National Defense Science and Engineering Graduate (NDSEG) Fellowship. The authors wish to thank Scott Meyer, Rob McGuire, BJ Austin, Nick Nugent, and Collin Morgan of Purdue University for their past and continued assistance.

\section{REFERENCES}

1. Culick, F., and V. Yang. 1995. Overview of combustion instabilities in liquidpropellant rocket engines. Liquid rocket engine combustion instability. Eds. W. An- 
derson and V. Yang. Progress in aeronautics and astronautics ser. Washington, DC. 169:3-37.

2. Sutton, G. P., and O. Biblarz. 2001. Rocket propulsion elements. 7th ed. New York, NY: John Wiley and Sons. $752 \mathrm{p}$.

3. Huang, X., and W. Baumann. 2007. Reduced-order modeling of dynamic heat release for thermoacoustic instability prediction. Combust. Sci. Technol. 179:617-636.

4. Krediet, H., W. Krebs, J. Portillo, and J. Kok. 2010. Prediction of thermoacoustic limit cycles during premixed combustion using the modified galerkin approach. 46th AIAA/ASME/SAE/ASEE Joint Propulsion Conference \& Exhibit. Nashville, TN.

5. Bellows, B., M. Bobba, J. Seitzman, and T. Lieuwen. 2007. Nonlinear flame transfer function characteristics in a swirl-stabilized combustor. J. Eng. Gas Turb. Power 129:954-961.

6. Palis, P., D. Durox, T. Schuller, and S. Candel. 2011. Nonlinear combustion instability analysis based on the flame describing function applied to turbulent premixed swirling flames. Combust. Flame 158:1980-1991.

7. Hield, P., M. Brear, and S. Jin. 2009. Thermoacoustic limit cycles in a premixed laboratory combustor with open and choked exits. Combust. Flame 156(9):1683-1697.

8. Culick, F. 1987. A note on Rayleigh's criterion. Combust. Sci. Technol. 56:159-166.

9. Thumuluru, S., H. Ma, and T. Lieuwen. 2007. Measurements of the flame response to harmonic excitation in a swirl combustor. 45th AIAA Aerospace Sciences Meetings and Exhibit. Reno, NV.

10. Paparizos, L., and F. Culick. 1986. The two-mode apporximation to nonlinear acoustics in combustion chambers I. Exact solution for second order acoustics. Combust. Sci. Technol. 65:39-65.

11. Pomeroy, B. 2012. Measurement and analysis of combustion response to transverse combustion instability. West Lafayette, IN: Purdue University, School of Aeronautics and Astronautics.

12. Holmes, P., G. Berkooz, and J. Lumley. 1996. Turbulence, coherent structures, dynamical systems and symmetry. Cambridge Monogr. Mech. Cambridge University Press.

13. Rowley, C., I. Mezic, P. Schaltter, and D. Henningson. 2009. Spectral analysis of nonlinear flows. J. Fluid Mech. 641:115-127.

14. Schmid, P. 2010. Dynamic mode decomposition of numerical and experimental data. J. Fluid Mech. 656:5-28.

15. Feldman, T., M. Harvazinski, C. Merkle, and W. Anderson. 2012. Comparison between simulation and measurement of self-excited combustion instability. 48th AIAA/ASME/SAE/ASEE Joint Propulsion Conference and Exhibit. Atlanta, GA.

16. Pomeroy, B., M. Wierman, and W. Anderson. 2013. Use of filtered combustion light and backlit high-speed images in combustion stability studies. Progr. Propul. Phys. 4:525-538. 